\title{
Knowledge and practices about hemodialysis among dialysis technicians in western part of India
}

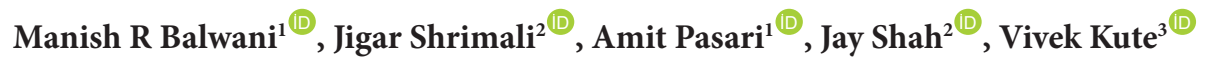 \\ ${ }^{1}$ Department of Nephrology, Jawaharlal Nehru Medical College, Sawangi, Wardha, Maharashtra, India \\ ${ }^{2}$ Department of Nephrology, Shalby Hospitals, Ahmedabad, India \\ ${ }^{3}$ Department of Nephrology, IKDRC \& ITS, BJMC, Ahmedabad, India
}

\section{A R T I C L E I N F O}

\section{Article Type:}

Original

\section{Article History:}

Received: 9 January 2019

Accepted: 23 May 2019

Published online: 28 June 2019

\section{Keywords:}

Knowledge

Practices

Hemodialysis technicians

Quality

Chronic kidney disease

End-stage renal disease

Hemodialysis

\begin{abstract}
A B S T R A C T
Introduction: Chronic kidney disease (CKD) is a global health burden with a high economic cost to health systems.

Objectives: To determine the knowledge and practices regarding end-stage renal disease and hemodialysis (HD) among dialysis technicians.

Patients and Methods: Total of 157 technicians participated in a study carried out at Ahmedabad city of Gujrat state. A validated written questionnaire was distributed among dialysis technicians who gave their consent to participate in the study and responded questionnaires were analyzed. Data entry was made in Excel software in codes and analysis was conducted by SPSS software version 17.0

Results: Out of 157, 113 participants were routinely using online clearance monitoring. Around, $19.1 \%$ carried out disinfection of water loop once per week, while $23.6 \%$ and $28 \%$ were doing it once in 15 days and 30 days respectively. Reverse osmosis (RO) plant parameters like $\mathrm{pH}$, hardness of water, chlorine content were checked once daily by $46.5 \%$ of technicians while $17.8 \%$ and $24.2 \%$ technicians were doing it once a week and once a month respectively. About $87.3 \%$ of technicians said that dialyzer was reused at their center. Accordingly, $33.8 \%$ and $51.6 \%$ of technicians were using formalin and per-acetic based disinfectants for dialyzer reprocessing. Additionally $7.6 \%$ of technicians were using hypochlorite as disinfectant at their center. Around $63.7 \%$ of technicians said they were not reprocessing dialyzers of HCV positive patients. They preferred to use single dialyzers in these subsets of patients at their center. Around, $79.6 \%$ of technicians said that endotoxin filter was installed in their dialysis unit. Likewise, $45.9 \%$ knew correctly the permissible level of endotoxin in RO water used for HD. Only 15.3\% of technicians had correct knowledge of permissible level of bacteria in treated $\mathrm{RO}$ water as per association for the advancement of medical instrumentation (AAMI) standards.

Conclusion: Better knowledge and awareness about dialysis standards among dialysis technicians will help in better patient's care. Effective dialysis education campaign needs to be driven at regular intervals with relevant information among dialysis technicians to address the knowledge gap.
\end{abstract}

Implication for health policy/practice/research/medical education:

Better knowledge and awareness about dialysis standards among dialysis technicians will help in better patient's care. Conducting regular educational meetings with dialysis technicians is vital for updating knowledge of technicians.

Please cite this paper as: Balwani MR, Shrimali J, Pasaril A, Shah J, Kute V. Knowledge and practices about hemodialysis among dialysis technicians in western part of India. J Nephropharmacol. 2020;9(1):e04. DOI: 10.15171/npj.2020.04.

Introduction

Chronic kidney disease (CKD) is a global health burden with a high economic cost to health systems. With increasing prevalence of diabetes mellitus, obesity and hypertension, the incidence of CKD and then end-stage renal disease (ESRD) will be rising. Kidney transplant being the best possible treatment for ESRD is not available to all, mainly due to shortage of organs. Thus, most of the
ESRD patients in India are on maintenance hemodialysis (MHD) twice to thrice per week. Apart from dialysis infrastructure and nephrologists, dialysis technicians play a vital role in providing good hemodialysis (HD) to patients.

\section{Objectives}

We tried to assess the knowledge and practices among HD 
technicians about ESRD and HD related aspects.

\section{Patients and Methods}

\section{Patient selection}

A cross-sectional study was conducted among HD technicians in a seminar organized at Ahmedabad city of Gujrat state of India. Among 200 participants, 157 consented to participate in the study. Prior approval of the institutional ethics committee was obtained. A validated written questionnaire was distributed among the participants. 20 minutes time was given to fill the questionnaire. Questionnaire was made keeping in mind various factors that were involved in $\mathrm{HD}$ practices. It focused on issues like use of online clearance monitoring (OCM), frequency of loop disinfection, checking of reverse osmosis (RO) plant parameters like $\mathrm{pH} /$ total dissolved solids (TDS)/chlorine content, reuse of dialyzer, chemical used for dialyzer reprocessing, use of endotoxin filter in water treatment plant, availability of full time nephrologist. It also assessed knowledge of technicians regarding RO water quality, association for the advancement of medical instrumentation (AAMI) standards, function of carbon filter, and shape of processed water storage tank and also hazards of high chlorine level in RO water.

\section{Ethics statement}

The study was conducted in accordance with the tenets of the Declaration of Helsinki. Prior approval of the institutional ethics committee was obtained. Informed consents from participants were also obtained.

\section{Statistical analysis}

Data entry was made in excel software in codes and analysis was done by SPSS software version 17.0. Descriptive statistical analysis, which included frequency, mean and percentages, was used to characterize the data.

\section{Results}

\section{Online clearance monitoring}

Out of 157, 113 (72\%) participants were routinely using online clearance monitoring while $42(26.8 \%)$ were not. Two of participants did not understand the online clearance monitoring meaning and have never heard about the same.

\section{Disinfection of RO water supply line}

Around $19.1 \%$ said they carry out disinfection of water loop once per week, while $23.6 \%$ and $28 \%$ were doing it once in 15 days and 30 days respectively. About $13.4 \%$ of technicians were doing it once in three months. Rest of the technicians did not answer the question.

\section{RO plant parameters}

$\mathrm{RO}$ plant parameters like $\mathrm{pH}$, hardness of water, chlorine content were checked once daily by $46.5 \%$ of technicians while $17.8 \%$ and $24.2 \%$ technicians were doing it once a week and once a month respectively. Rest of technicians had no idea about the frequency of checking of these parameters at their unit.

\section{Dialyzer reuse and reprocessing}

Around $87.3 \%$ of technicians said that dialyzer was reused at their center. $33.8 \%$ and $51.6 \%$ technicians were using formalin and per-acetic based disinfectants for dialyzer reprocessing $7.6 \%$ technicians were using hypochlorite as disinfectant at their center.

\section{HCV positive patients}

About $63.7 \%$ of technicians said they were not reprocessing dialyzers of $\mathrm{HCV}$ positive patients. They preferred to use single-use dialyzers in these subsets of patients at their center.

Similarly $68.2 \%$ of technicians opined about the use of single use dialyzer in $\mathrm{HbsAg}$ positive patients at their center where they did not prefer to reprocess the dialyzer in these subsets of patients.

\section{Endotoxin filter}

About $79.6 \%$ of technicians said that endotoxin filter was installed in their dialysis unit.

\section{Availability of nephrologist}

Around $45.2 \%$ of technicians informed that their dialysis facility did not have fulltime nephrologist. Accordingly, $72 \%$ of technicians informed about the presence of round the clock either MBBS or MD doctor in their dialysis unit.

\section{AAMI standards}

About $45.9 \%$ knew correctly the permissible level of endotoxin in RO water used for $\mathrm{HD}$, while $25.5 \%$ had no idea about the AAMI standards. Rest of technicians had incorrect knowledge about the AAMI standards. However, only $15.3 \%$ of technicians had correct knowledge of permissible level of bacteria in treated RO water as per AAMI standards.

\section{Carbon filter}

About $24.8 \%, 36.9 \%$ and $19.1 \%$ of technicians respectively said that 1, 2 and 3 carbon filters should be installed in a typical water treatment unit for HD, while, the rest had no idea regarding same.

Only $40.8 \%$ of technicians knew that carbon filter is mainly used for removal of chlorine from the water. Since, $22.3 \%$ of technicians had a wrong impression that it is mainly used for removal of hardness of water. In addition, $17.2 \%$ of technicians thought that its main function is to remove bacteria from the water, since, rest had no idea about the exact function of carbon filter.

\section{Shape of water storage tank}

About $30.6 \%$ of technicians opined that shape of tank used for processed RO water should have conical bottom, 
since $22.9 \%$ of technicians remarked that it should have flat bottom and $12.7 \%$ expressed the shape actually does not matter, while the rest of them did not have any idea about it.

\section{Chlorine level}

About 26.8\% of technicians expressed that chlorine level of processed water should be checked daily while $36.9 \%$ and $17.2 \%$ highlighted it should be checked weekly and monthly respectively, while the rest of them had no knowledge regarding it. However, $54.1 \%$ correctly opined about hemolytic side effect of high chlorine level in processed water used for HD.

\section{Fistula care}

Around $78.3 \%$ of technicians usually ask the patients to wash the fistula arm prior to start of dialysis, since $65 \%$ technicians ask their patients to wash the fistula arm with antibacterial soap prior to onset of dialysis while $10.2 \%$ and $5.1 \%$ ask their patients to wash off their fistula arm with simple water and ordinary soap respectively, while the rest of technicians do not routinely ask their patients to wash the fistula arm prior to onset of HD.

\section{Discussion}

HD technicians are one of the key persons for successful running of HD program. Regular update of knowledge is critical to bring forth the best quality practices in any profession as is with HD. In this study, we tried to assess the practices adopted by dialysis technicians at various centers and their knowledge regarding $\mathrm{RO}$ water and also HD. This kind of study has never been carried out in our region, therefore we thought to go ahead with this study so that we can appropriately target the study content to be shared among dialysis technicians in further educational programs. This study has its own limitations as it is based on the knowledge of technicians and may not necessarily reflect the exact pattern of practice being carried out at the dialysis centers.

HD dose has direct impact upon the morbidity and mortality rate in patients on regular dialysis therapy. Accordingly, the adequacy of dialysis treatment should be given with regular monitoring of online clearance. In our study, $72 \%$ of dialysis technicians were regularly using online clearance monitoring. Most of the centers now have HD machines equipped with online clearance monitoring $\left(\mathrm{OCM}^{\circledR}\right)$, where it can be easily monitored. The OCM option of the HD machine provides a safe and accurate tool for continuous online monitoring of total urea clearance. The online clearance tool can be of great help to improve the quality of life of patients achieving adequate clearance.

Patients undergoing HD 'three times per week' can be exposed to 300-600 liter of water every week depending on their prescription $(1,2)$. The volume of dialysis fluid increases for those on nocturnal treatments to 580-860 1 per week (2). Ensuring the necessary quality of dialysate is a vital aspect of HD treatment since each patient is exposed to particularly bacteria, and associated endotoxin contamination which can threaten a dialysis patient's health.

Water is treated, purified, and transported through a distribution system within a dialysis center where it is used in the preparation of dialysate concentrates, as well as for proportioning concentrates at the dialysis machine to produce the final dialysate. At all of these steps, there is possibility for microbial growth or chemical exposure if the water is contaminated and not properly maintained.

The dialysis staff must be cognizant of their incoming water quality and the provider's treatment practices prior to beginning dialysis preparation and dialysis treatment.

The 2009 and 2011 updated recommendations by AAMI are more stringent and are voluntary $(3,4)$. All of these guidelines and recommendations focus on the quality management of dialysis treatment, of which water or dialysate are the main topics. These recommendations are necessary to be followed due to the potential health outcomes for dialysis patients if exposed to chemical or microbiological contaminants.

For example, sulfate $(>200 \mathrm{mg} / \mathrm{L})$ is associated with nausea, vomiting, and metabolic acidosis (5), while lead $(52-65 \mu \mathrm{g} / \mathrm{L})$ has caused abdominal pain and muscle weakness (6).

Microbial contaminated dialysis water and/or dialysate can produce bacteremia and chronic inflammation, which contributes to increased morbidity and mortality.

Endotoxin fragments in the dialysate bath can pass through the dialyzer membranes and stimulate circulating immune cells to produce symptoms of septicemia or a pyrogenic reaction. It can also trigger inflammatory markers, such as high-sensitivity C-reactive protein, interleukin (IL)-6, fibrinogen, and intercellular adhesion molecule (sICAM-1) (7).

Chronic inflammation contributes to cardiovascular disease (CVD) and also has been linked to the poor nutritional status, reduced response to erythropoietin therapy, decline in residual renal function and carpal tunnel syndrome (8).

The 2011 AAMI recommendations lowered the acceptable endotoxin concentration to $<0.25 \mathrm{EU} / \mathrm{ml}$ in dialysis water and $<0.5 \mathrm{EU} / \mathrm{ml}$ in the dialysate (4). Ultrapure dialysis requires the endotoxin concentration in the dialysate to be $<0.03 \mathrm{EU} / \mathrm{mL}$. In this study, $45.9 \%$ knew correctly the permissible level of endotoxin in RO water used for HD. In our study, $25.5 \%$ had no idea about the AAMI standards, since, the rest of technicians had incorrect knowledge about the AAMI standards. Only $15.3 \%$ of technicians had correct knowledge of permissible level of bacteria in treated RO water as per AAMI standards

Validation of a dialysis system and its monitoring is crucial for establishing that the system can both provide 
the necessary water quality and whether the disinfection processes are sufficient at keeping the microbial contaminants below the maximum allowable limits.

The chemical contaminants within the water system are to be tested at least annually in combination with the evaluation of the source water (incoming feed water) (4).

As per AAMI standards, total chlorine should be monitored prior to each patient shift after the primary carbon tank to confirm that the concentration is below $0.01 \mathrm{mg} / \mathrm{L}$ (4). In our study, RO plant parameters like $\mathrm{PH}$, hardness of water, chlorine content were checked once daily by $46.5 \%$ of technicians while $17.8 \%$ and $24.2 \%$ of technicians were doing it once a week and once a month respectively. The rest of technicians had no idea about the frequency of checking of these parameters at their unit. Only $40.8 \%$ of technicians knew that carbon filter is mainly used for removal of chlorine from the water, since $22.3 \%$ of technicians had a wrong impression that it is mainly used for removal of hardness of water. However, $17.2 \%$ of technicians thought that its main function is to remove bacteria from the water. Rest of them had no idea about the exact function of carbon filter. Accordingly, $26.8 \%$ technicians said that chlorine level of processed water should be checked daily while $36.9 \%$ and $17.2 \%$ said it should be checked weekly and monthly respectively, since rest of them had no knowledge regarding same. In addition, 54.1\% correctly opined about hemolytic side effect of high chlorine level in processed water used for HD.

The dialysis storage water tanks and water distribution piping system should be monitored once a month, or as determined from the validation process, for microorganisms and endotoxin (4). In our study, 19.1\% said they carry out disinfection of water loop once per week, while $23.6 \%$ to $28 \%$ were doing it once in 15 days and 30 days respectively. However, $13.4 \%$ of technicians were doing it once in three months, since, the rest of the technicians did not answer the question.

The standard dialysis fluid from each dialysis machine within a facility should be tested at least once a year for bacteria and endotoxin, where regular testing is conducted on a different machine each month (machines are tested on a rotation). Ultrapure dialysis fluid is also tested monthly, but only for endotoxin. However, if the bacteria- and endotoxin-retentive filter is validated, operated, and monitored according to the manufacturer's instructions, this testing may not be necessary. Specifically for endotoxin-retentive filters, daily monitoring of the pressure across the filter is adequate in assuring endotoxin levels are within the limit. In our study, 79.6\% of technicians said that endotoxin filter was installed in their dialysis unit.

Additionally, for anyone who develops signs and symptoms of bacteremia during the dialysis session, blood cultures and dialysate (from the patient's machine) for cultures and endotoxin should be obtained as a routine part of the patient workup.

A significant finding is that the reuse of dialyzers has been associated with $50 \%$ of the microbial-associated outbreaks (9-13). In our study, $87.3 \%$ of technicians said that dialyzer was reused at their center. Accordingly $33.8 \%$ and $51.6 \%$ of technicians were using formalin and per-acetic based disinfectants for dialyzer reprocessing. Around $7.6 \%$ of technicians were using hypochlorite as disinfectants at their center. Reprocessing or reuse of dialyzers renders the dialyzers vulnerable to contamination from water used for rinsing, inadequate disinfection, and potential alterations to the permeability of the membrane. In our study, $63.7 \%$ of technicians said they were not reprocessing dialyzers of HCV positive patients. They preferred to use singleuse dialyzers in these subsets of patients at their center. Similarly $68.2 \%$ of technicians opined about the use of single use dialyzer in $\mathrm{HbsAg}$ positive patients at their center where they did not prefer to reprocess the dialyzer in these subsets of patients.

In our study, $45.2 \%$ of technicians informed that their dialysis facility did not have fulltime nephrologist. While $72 \%$ of technicians informed about the presence of round the clock either MBBS or MD doctor in their dialysis unit. With increasing availability of nephrologists, probably this problem will be solved in the coming years.

Water storage and distribution systems contain large amounts of processed water. The larger volume and surface area increases the potential for biofilm formation. The tank should be designed to minimize the growth of bacteria by having a conical or bowl-shaped bottom to allow for complete emptying. About $30.6 \%$ of technicians opined that shape of tank used for processed RO water should have conical bottom. About $22.9 \%$ of technicians said that it should have flat bottom and $12.7 \%$ highlighted the shape actually does not matter, since rest of them said that they do not have any idea about the same.

Patients with poor hygiene were shown to have a significantly increased incidence of skin colonization of the access site with staph aureus after skin preparation, thus poor hygiene is a major risk factor for the development of access site infections (14). In our study, $78.3 \%$ of technicians usually ask the patients to wash the fistula arm prior to start of dialysis. Around $65 \%$ of technicians ask their patients to wash the fistula arm with antibacterial soap prior to onset of dialysis while $10.2 \%$ and $5.1 \%$ ask their patients to wash off their fistula arm with simple water and ordinary soap respectively. Rest of the technicians do not routinely ask their patients to wash the fistula arm prior to onset of HD.

\section{Conclusion}

Conducting regular educational meetings with dialysis technicians is vital for updating knowledge of technicians. Our study shows significant gap in recommendations and 
prevalent practices. With increasing number of patients reaching ESRD, need of adequately trained HD technicians will increase further. Authors suggest that HD technicians should undergo continuous medical education programs related to dialysis at regular intervals since there is a need of central licensing authority to monitor their skills and knowledge.

\section{Study limitations}

Limited sample size is the limitation of the study.

Authors' contribution

All authors wrote the paper equally.

\section{Conflicts of interest}

The authors declare that they have no conflicts interest.

\section{Ethical considerations}

Ethical issues (including plagiarism, data fabrication, double publication) have been completely observed by the authors.

\section{Funding/Support}

None.

\section{References}

1. AAMI. TIR43: ultrapure dialysate for hemodialysis and related therapies. Arlington, VA: Association for the Advancement of Medical Instrumentation; 2011. [May 30, 2019] Available from: http://my.aami.org/aamiresources/ previewfiles/TIR431109_preview.pdf.

2. Ward RA. Avoiding toxicity from water-borne contaminants in hemodialysis: new challenges in an era of increased demand for water. Adv Chronic Kidney Dis. 2011;18:20713. doi: 10.1053/j.ackd.2011.01.007.

3. ANSI/AAMI/ISO. Quality of dialysis fluid for hemodialysis fluid and related therapies 11663:2009.
Arlington, VA: Association for the Advancement of Medical Instrumentation; 2009. [May 30, 2019] Available from: https://www.techstreet.com/mss/products/ preview/1765246.

4. ANSI/AAMI/ISO. Guidance for the preparation and quality management of fluids for hemodialysis and related therapies 23500:2011. Arlington, VA: Association for the Advancement of Medical Instrumentation; 2011.

5. .Comty C, Luehmann D, Wathen R, Shapiro F. Prescription water for chronic hemodialysis. Trans Am Soc Artif Intern Organs. 1974; 20A:189-96.

6. Kathuria P, Nair B, Schram D, Medlock R. Outbreak of lead poisoning in a hemodialysis unit. J Am Soc Nephrol. 2004; 15:617A.

7. Stenvinkel P, Alvestrand A. Inflammation in end-stage renal disease: sources, consequences, and therapy. Semin Dial. 2002;15:329-37.

8. Ward RA. Ultrapure dialysate. Semin Dial. 2004;17:489-97. doi: 10.1111/j.0894-0959.2004.17617.x.

9. CDC Clusters of bacteremia and pyrogenic reactions in hemodialysis patients - Georgia (EPI-86-65-2). Epidemic Investigations Reports. 1987.

10. Welbel SF, Schoendorf K, Bland LA, Arduino MJ, Groves C, Schable B, et al. An outbreak of gram-negative bloodstream infections in chronic hemodialysis patients. Am J Nephrol. 1995;15:1-4. doi: 10.1159/000168793.

11. Gordon SM, Tipple M, Bland LA, Jarvis WR. Pyrogenic reactions associated with the reuse of disposable hollowfiber hemodialyzers. JAMA. 1988;260:2077-81.

12. CDC Bacteremia associated with reuse of disposable hollowfiber dialyzers (EPI-86-44-2). Epidemic Investigations Report. 1986.

13. CDC Pyrogenic reactions in hemodialysis patients on highflux hemodialysis - California (EPI-87-12-2). Epidemic Investigations Report. 1987.

14. Kaplowitz LG, Comstock JA, Landwehr DM, Dalton HP, Mayhall CG. Prospective study of microbial colonization of the nose and skin and infection of the vascular access site in hemodialysis patients. J Clin Microbiol. 1988;26:1257-62.

Copyright (c) 2020 The Author(s); Published by Published by Society of Diabetic Nephropathy Prevention. This is an open-access article distributed under the terms of the Creative Commons Attribution License (http://creativecommons.org/licenses/by/4.0), which permits unrestricted use, distribution, and reproduction in any medium, provided the original work is properly cited. 